\title{
ON REAL TRIGONAL RIEMANN SURFACES
}

\author{
ANTONIO F. COSTA and MILAGROS IZQUIERDO*
}

\begin{abstract}
A closed Riemann surface $X$ which can be realized as a 3-sheeted covering of the Riemann sphere is called trigonal, and such a covering will be called a trigonal morphism. A trigonal Riemann surface $X$ is called real trigonal if there is an anticonformal involution (symmetry) $\sigma$ of $X$ commuting with the trigonal morphism. If the trigonal morphism is a cyclic regular covering the Riemann surface is called real cyclic trigonal. The species of the symmetry $\sigma$ is the number of connected components of the fixed point set $\operatorname{Fix}(\sigma)$ and the orientability of the Klein surface $X /\langle\sigma\rangle$. We characterize real trigonality by means of Fuchsian and NEC groups. Using this approach we obtain all possible species for the symmetry of real cyclic trigonal and real non-cyclic trigonal Riemann surfaces.
\end{abstract}

\section{Introduction}

A closed Riemann surface $X$ which can be realized as a 3-sheeted covering of the Riemann sphere is said to be trigonal, and such a covering will be called a trigonal morphism. Trigonal surfaces have been recently studied, see [3] and [11]. A trigonal Riemann surface $X$ is called real trigonal if there is an anticonformal involution $\sigma$ of $X$ commuting with the trigonal morphism. For instance, if $X$ is represented by a curve given by the complex solutions of a polynomial equation with real coefficients of the form: $y^{3}+y b(x)+c(x)=0$, then $X$ is a real trigonal Riemann surface.

If the trigonal morphism is a cyclic regular (cyclic-Galois) covering, i.e. its deck-transformations group acts transitively on the preimage of each point and it is a cyclic group, then the Riemann surface is called real cyclic trigonal.

Given a real trigonal Riemann surface $X$ of genus $g$ the topological type of the action of the anticonformal involution $\sigma$, i.e. the topological type of the quotient orbifold $X /\langle\sigma\rangle$, is determined by the number of connected components, called ovals, of the fixed point set $\operatorname{Fix}(\sigma)$ and the orientability of the Klein surface $X /\langle\sigma\rangle$. We say that $\sigma$ has species $+k$ if $\operatorname{Fix}(\sigma)$ consists of $k$ ovals and $X /\langle\sigma\rangle$ is orientable, and $-k$ if $\operatorname{Fix}(\sigma)$ consists of $k$ ovals and $X /\langle\sigma\rangle$

\footnotetext{
* The first named author is partially supported by BFM2002-04801. The second named author is partially supported by the Swedish Natural Science Research Council (NFR) and The Swedish Foundation for International Cooperation in Research and Higher Education (STINT)

Received February 2, 2004; in revised form June 29, 2005.
} 
is nonorientable (i.e. two surfaces with symmetries of the same species have topologically conjugate quotients and vice versa). The set Fix $(\sigma)$ corresponds to the real part of a complex algebraic curve representing $X$, which admits an equation with real coefficients. The " + " sign in the species of $\sigma$ means that the real part disconnects its complement in the complex curve and then we say that $\sigma$ separates. By a classical theorem of Harnack the possible values of species run between $-g$ and $+(g+1)$, where $+k \equiv g+1(\bmod 2)$ (see [6]). F. Klein, in [10], obtained the possible species of hyperelliptic Riemann surfaces, double coverings of the Riemann sphere. For surfaces that are double coverings of other surfaces the study has been done in [5]. In [7], Section 8, Gross and Harris study the species of real trigonal surfaces (3-sheeted covering of the sphere) and they ask for the possible values for the species of some special types of real trigonal surfaces.

In Section 2 we characterize real trigonality by means of Fuchsian and NEC groups. Using this approach we obtain all possible species for real cyclic trigonal and real non-cyclic trigonal Riemann surfaces. More concretely, in Section 3 the possible species for real cyclic trigonal Riemann surfaces are calculated: they are -1 for all genera, and also +3 and +1 for even genera. The species +3 and +1 , for the Case 2 in the proof of Theorem 3.1, correspond to cases (2) and (1) in page 179 of [7] (three real components of degree 1 over $\mathrm{P}^{1}(\mathrm{R})$ and one real component of degree 3 over $\mathrm{P}^{1}(\mathrm{R})$ ); the species -1 and +1 , for the Case 1 in Theorem 3.1, correspond to curves with one real component of degree 1 over $P^{1}(R)$.

In Theorem 4.1 of Section 4 we show that the only restrictions on the species of real non-cyclic trigonal Riemann surfaces are given by the theorem of Harnack. The surfaces constructed in Theorem 4.1 correspond to real trigonal curves of type (4) in [7], answering the question raised by Gross and Harris.

AcKnowledgment. The authors are grateful to Emilio Bujalance, Göran Bergqvist and Peter Turbek for helpful revision of the preliminary version of this paper.

\section{Trigonal Real Riemann surfaces and Fuchsian and NEC groups}

Let $X_{g}$ be a compact Riemann surface of genus $g \geq 2$. The surface $X_{g}$ can be represented as a quotient $X_{g}=\mathscr{D} / \Gamma$ of the complex unit disc $\mathscr{D}$ under the action of a Fuchsian group $\Gamma$, that is, a cocompact orientation-preserving subgroup of the group $\mathscr{G}=\operatorname{Aut}(\mathscr{D})$ of conformal and anticonformal automorphisms of $\mathscr{D}$. A discrete, cocompact subgroup $\Gamma$ of $\operatorname{Aut}(\mathscr{D})$ is called an NEC (non-euclidean crystallographic) group. The subgroup of $\Gamma$ consisting of the orientation-preserving elements is called the canonical Fuchsian subgroup of $\Gamma$. The algebraic structure of an NEC group and the geometric and 
topological structure of its quotient orbifold are given by the signature of $\Gamma$ :

(1) $s(\Gamma)=\left(h ; \pm ;\left[m_{1}, \ldots, m_{r}\right] ;\left\{\left(n_{11}, \ldots, n_{1 s_{1}}\right), \ldots,\left(n_{k 1}, \ldots, n_{k s_{k}}\right)\right\}\right)$.

The orbit space $\mathscr{D} / \Gamma$ is an orbifold with underlying surface of genus $h$, having $r$ cone points and $k$ boundary components, each with $s_{j} \geq 0$ corner points, $j=$ $1, \ldots, k$. The signs ' + ' and ' - ' correspond to orientable and non-orientable orbifolds respectively. The integers $m_{i}$ are called the proper periods of $\Gamma$ and they are the orders of the cone points of $\mathscr{D} / \Gamma$. The brackets $\left(n_{i 1}, \ldots, n_{i s_{i}}\right)$ are the period cycles of $\Gamma$. The integers $n_{i j}$ are the link periods of $\Gamma$ and the orders of the corner points of $\mathscr{D} / \Gamma$. The group $\Gamma$ is called the fundamental group of the orbifold $\mathscr{D} / \Gamma$. We shall use the notation $m^{u}$ for $m, . . ., m$ in signatures $(u$ may be 0 ).

A group $\Gamma$ with signature (1) has a canonical presentation with four types of generators:

1. Hyperbolic generators $a_{1}, b_{1}, \ldots, a_{h}, b_{h}$ if $\mathscr{D} / \Gamma$ is orientable or glide reflection generators: $d_{1}, \ldots, d_{h}$ if $\mathscr{D} / \Gamma$ is non-orientable,

2. Elliptic generators: $x_{1}, \ldots, x_{r}$

3. Connecting generators (hyperbolic or elliptic transformations): $e_{1}, \ldots, e_{k}$

4. Reflection generators: $c_{i j}, 1 \leq i \leq k, 1 \leq j \leq s_{i}+1$.

And relators:

1. $x_{i}^{m_{i}}, i=1, \ldots, r$,

2. $c_{i j}^{2}$,

3. $\left(c_{i j-1} c_{i j}\right)^{n_{i j}}$,

4. $c_{i 0} e_{i}^{-1} c_{i s_{i}} e_{i}, i=1, \ldots, k, j=2, \ldots, s_{i}+1$,

5. The long relation: $x_{1} \ldots x_{r} e_{1} \ldots e_{k} a_{1} b_{1} a_{1}^{-1} b_{1}^{-1} \ldots a_{h} b_{h} a_{h}^{-1} b_{h}^{-1}$ or $x_{1} \ldots x_{r} e_{1} \ldots e_{k} d_{1}^{2} \ldots d_{h}^{2}$, according to whether $\mathscr{D} / \Gamma$ is orientable or not.

The hyperbolic area of the orbifold $\mathscr{D} / \Gamma$ coincides with the hyperbolic area of an arbitrary fundamental region of $\Gamma$ and equals:

$$
\mu(\Gamma)=2 \pi\left(\varepsilon h-2+k+\sum_{i=1}^{r}\left(1-\frac{1}{m_{i}}\right)+\frac{1}{2} \sum_{i=1}^{k} \sum_{j=1}^{s_{i}}\left(1-\frac{1}{n_{i j}}\right)\right),
$$

where $\varepsilon=2$ if there is a " + " sign and $\varepsilon=1$ otherwise. If $\Gamma^{\prime}$ is a subgroup of $\Gamma$ of finite index then $\Gamma^{\prime}$ is an NEC group and the following Riemann-Hurwitz formula holds:

$$
\left[\Gamma: \Gamma^{\prime}\right]=\mu\left(\Gamma^{\prime}\right) / \mu(\Gamma)
$$


The orbit space $\mathscr{D} / \Gamma$ is a Klein or Riemann surface, i.e. a surface with a dianalytic structure of topological genus $h$, orientable or not according to the sign "+" or "-", and possibly having $k$ boundary components and we say that $\Gamma$ uniformizes $\mathscr{D} / \Gamma$.

An NEC or Fuchsian group $\Gamma$ without elliptic elements is called an NEC or Fuchsian surface group and it has signature $(h ; \pm ;[-],\{(-), . k .,(-)\})$ or $(h ;[-])$. Given a Riemann (resp. Klein) surface represented as the orbit space $X=\mathscr{D} / \Gamma$, with $\Gamma$ a Fuchsian (resp. NEC) surface group, a finite group $G$ is a group of automorphisms of $X$ if and only if there exists an NEC group $\Delta$ and an epimorphism $\theta: \Delta \rightarrow G$ with $\operatorname{ker}(\theta)=\Gamma$. The NEC group $\Delta$ is the lifting of $G$ to the universal covering $\pi: \mathscr{D} \rightarrow \mathscr{D} / \Gamma$ and is called the universal covering transformations group of $(X, G)$.

Definition 2.1. A trigonal Riemann surface is a pair $(X, f)$, where $f$ : $X \rightarrow \widehat{\mathrm{C}}$ is a 3-sheeted covering from the Riemann surface $X$ onto the Riemann sphere. If $f$ is a cyclic regular covering then $(X, f)$ is called cyclic trigonal. The covering map $f$ will be called the cyclic (resp. non-cyclic) trigonal morphism.

By [1] and Lemma 2.1 in [2], if the surface $X$ has genus $g \geq 5$, then the trigonal morphism is unique, hence any automorphism of $X$ must commute with the trigonal morphism. From now on we shall consider surfaces with genera greater than 4 , and then we shall identify the pair $(X, f)$ with the surface $X$.

THEOREM 2.2. A Riemann surface $X$ admits a cyclic trigonal morphism $f$ if and only if there is a Fuchsian group $\Delta^{+}$with signature

$$
\left(0,\left[3^{g+2}\right]\right)
$$

and an index three normal surface subgroup $\Gamma$ of $\Delta^{+}$, such that $\Gamma$ uniformizes $X$.

We are using the notation $3^{g+2}$ for $3, \stackrel{g+2}{.}, 3$.

Proof. Let $(X, f)$ be a cyclic trigonal surface. Then there is an order three automorphism $\varphi: X \rightarrow X$ such that $X /\langle\varphi\rangle$ is the Riemann sphere and $\varphi$ is a deck-transformation of the covering map $f$. Let $\Gamma$ be a Fuchsian surface group uniformizing $X$ and let $\widetilde{\varphi}$ be a lifting of $\varphi$ to the universal covering $\mathscr{D} \rightarrow \mathscr{D} / \Gamma=X$. We call $\Delta^{+}=\langle\Gamma, \widetilde{\varphi}\rangle$ the universal covering transformations group of $(X,\langle\varphi\rangle)$. Since $f: \mathscr{D} / \Gamma \rightarrow \mathscr{D} / \Delta^{+}$is a 3 -sheeted regular covering, by the Riemann-Hurwitz formula the signature of $\Delta^{+}$is $\left(0,\left[3^{g+2}\right]\right)$ and $\Gamma$ is an index three normal surface subgroup of $\Delta^{+}$.

Let now $\Delta^{+}$be a Fuchsian group with signature $\left(0,\left[3^{g+2}\right]\right)$ and $\Gamma$ an index three normal surface subgroup of $\Delta^{+}$, such that $X=\mathscr{D} / \Gamma$. Then $f: X=$ 
$\mathscr{D} / \Gamma \rightarrow \mathscr{D} / \Delta^{+}$is a cyclic trigonal morphism and $(X, f)$ is a cyclic trigonal surface.

THEOREM 2.3. A Riemann surface $X$ of genus $g$ admits a non-cyclic trigonal morphism $f$ if and only if there is a Fuchsian group $\Delta^{+}$with signature

$$
\left(0,\left[2^{u}, 3^{v}\right]\right) \quad \text { where } u+2 v=2 g+4, u \equiv 0(\bmod 2), u \neq 0,
$$

and an index three non-normal subgroup $\Gamma$ of $\Delta^{+}$, with signature $\left(g,\left[2^{u}\right]\right)$ such that $\mathscr{D} / \Gamma$ is conformally equivalent to $X$.

Proof. Let $(X, f)$ be a non-cyclic trigonal surface, and let $f: X \rightarrow \widehat{\mathrm{C}}$ be the trigonal morphism, this is a 3-sheeted branched covering map. Let $B$ be the set of branch points of $f$. The monodromy of $f$ is $\omega: \pi_{1}(\widehat{C}-B) \rightarrow \Sigma_{3}$, where $\Sigma_{3}$ is the symmetric group of three elements $\{0,1,2\}$ and $f_{*}\left(\pi_{1}(X-\right.$ $\left.\left.f^{-1}(B)\right)\right)=\omega^{-1}(\operatorname{Stab}(0))$, where $0,1,2$ are the labels of the three sheets of the covering $f$. See Chapter 8 in [12] for a classical reference about monodromy and coverings. Then ker $\omega$ defines a regular covering $\widetilde{f}: \widetilde{X} \rightarrow \widehat{C}$, branched on $B$ and with deck-transformations group $\Sigma_{3}$. Thus there is an element $t$ of order two in $\Sigma_{3}$ such that $\widetilde{X} \rightarrow \widetilde{X} /\langle t\rangle=X \stackrel{f}{\rightarrow} \widetilde{X} / \Sigma_{3}=\widehat{C}$. Hence there is a Fuchsian group $\Delta^{+}$, the universal covering transformations group of $\left(X, \Sigma_{3}\right)$, and an epimorphism $\theta: \Delta^{+} \rightarrow \Sigma_{3}$, such that $\operatorname{ker} \theta$ is a Fuchsian surface group uniformizing $\widetilde{X}$. Now $X$ is uniformized by the subgroup $\theta^{-1}(\langle t\rangle)=\Gamma$ of $\Delta^{+}$with index three. The elements of $\Sigma_{3}$ have orders 1,2 or 3 , then $\Delta^{+}$has signature $\left(0,\left[2^{u}, 3^{v}\right]\right)$ and, since $\widetilde{X}=\mathscr{D} / \operatorname{ker} \theta \rightarrow \mathscr{D} / \Gamma=X$ is a double covering, the signature of $\Gamma$ is $\left(g,\left[2^{u}\right]\right)$. The condition $u+2 v=2 g+4$ follows from Riemann-Hurwitz formula.

Assume now that we have a Fuchsian group $\Delta^{+}$with signature (5) and an index three non-normal subgroup $\Gamma$ of $\Delta^{+}$, with signature $\left(g,\left[2^{u}\right]\right)$ such that $\mathscr{D} / \Gamma$ is conformally equivalent to $X$. The meromorphic function $f: \mathscr{D} / \Gamma \rightarrow$ $\mathscr{D} / \Delta^{+}$is a trigonal morphism and $(X, f)$ is a non-cyclic trigonal surface.

Definition 2.4. A real Riemann surface is a pair $(X, \sigma)$, where $X$ is a Riemann surface and $\sigma: X \rightarrow X$ is an anticonformal involution (symmetry) of $X$. A real trigonal Riemann surface is a triple $(X, f, \sigma)$ where $(X, \sigma)$ is a real Riemann surface, $(X, f)$ is a trigonal Riemann surface and $f \circ \sigma=c \circ f$, with $c$ the complex conjugation.

By [1] the condition $f \circ \sigma=c \circ f$ is automatically satisfied for genera $g \geq 5$.

We give now a characterization of real cyclic trigonal surfaces via NEC groups. 
THEOREM 2.5. Let $X$ be a Riemann surface with genus $g \geq 5$. The surface $X$ admits a symmetry $\sigma$ and a meromorphic function $f$ such that $(X, f, \sigma)$ is a real cyclic trigonal Riemann surface if and only if there is an NEC group $\Delta$ with signature

$$
\left(0,+,\left[3^{u}\right],\left\{\left(3^{v}\right)\right\}\right)
$$

where $2 u+v=g+2$, such that there is an epimorphism $\theta: \Delta \rightarrow G$, with $G$ isomorphic to either $D_{3}=\left\langle r, s: r^{3}=s^{2}=r s r s=1\right\rangle$ or $\mathrm{Z}_{6}=\left\langle r, s: r^{3}=\right.$ $\left.s^{2}=r s r^{-1} s=1\right\rangle$, where $X$ is conformally equivalent to $D / \operatorname{ker} \theta$ and $\operatorname{ker} \theta$ is a Fuchsian surface group.

Proof. Let $(X, f, \sigma)$ be a real cyclic trigonal surface. Then there is an order three automorphism $\varphi: X \rightarrow X$ such that $X /\langle\varphi\rangle$ is the Riemann sphere and $\varphi$ is a deck-transformation of the covering $f$. Let $\Gamma$ be a Fuchsian surface group uniformizing $X, \tilde{\varphi}$ being a lifting of $\varphi$ to the universal covering $\mathscr{D} \rightarrow \mathscr{D} / \Gamma=$ $X$ and $\widetilde{\sigma}$ being the lifting of $\sigma$. Since $f \circ \sigma=c \circ f, \widetilde{\sigma}$ is a lifting of $c$ on $\widehat{\mathrm{C}}$ by the chain of coverings $\mathscr{D} \rightarrow \mathscr{D} / \Gamma=X \stackrel{f}{\rightarrow} \widehat{\mathrm{C}}$. We set $\Delta=\langle\Gamma, \widetilde{\varphi}, \widetilde{\sigma}\rangle$ and $\Delta^{+}=\langle\Gamma, \widetilde{\varphi}\rangle$. Note that $\Delta^{+}$is the canonical Fuchsian group of $\Delta$. Since $f: \mathscr{D} / \Gamma \rightarrow \mathscr{D} / \Delta^{+}$is a 3 -sheeted regular covering, the signature of $\Delta^{+}$is $\left(0,\left[3^{g+2}\right]\right)$ and the signature of $\Delta$ is (6). Moreover $G=\langle\varphi, \sigma\rangle$ is $D_{3}$ or $Z_{6}$ because the trigonal morphism is unique and then $\sigma \varphi \sigma=\varphi^{ \pm 1}$. The action of $\Delta$ on the $\Gamma$-cosets gives us the required epimorphism $\theta: \Delta \rightarrow G$.

Let $\Delta$ be an NEC group with signature (6) such that there is an epimorphism $\theta: \Delta \rightarrow G$, where $G$ is isomorphic to either $D_{3}=\left\langle r, s: r^{3}=s^{2}=r s r s=1\right\rangle$ or $\mathrm{Z}_{6}=\left\langle r, s: r^{3}=s^{2}=r s r^{-1} s=1\right\rangle$, with $\operatorname{ker} \theta$ a Fuchsian surface group. Let $X$ be the surface uniformized by $\operatorname{ker} \theta$. Then the canonical Fuchsian group $\Delta^{+}$of $\Delta$ has signature (4) and the meromorphic function $f: X=\mathscr{D} / \Gamma \rightarrow$ $\mathscr{D} / \Delta^{+}$is a cyclic trigonal morphism. The quotient $X=\mathscr{D} / \Gamma \rightarrow \mathscr{D} / \theta^{-1}(\langle s\rangle)$ provides a symmetry $\sigma$ of $X$ in such a way that $(X, f, \sigma)$ is a real cyclic trigonal surface.

We give now a characterization of real non-cyclic trigonal surfaces via NEC groups.

THeOREM 2.6. Let $X$ be a Riemann surface of genus $g \geq 5$. The surface $X$ admits a symmetry $\sigma$ and a meromorphic function $f$ such that $(X, f, \sigma)$ is a real non-cyclic trigonal Riemann surface if and only if there is an NEC group $\Delta$ with signature

$$
\left(0,+,\left[3^{u_{1}}, 2^{u_{2}}, 4^{u_{3}}\right],\left\{\left(3^{v_{1}}, 2^{v_{2}}, 4^{v_{3}}, 3^{v_{4}}, 2^{v_{5}}, \ldots\right)\right\}\right), u_{2}+\sum_{i} v_{3 i+2}>0
$$


such that there is an epimorphism $\theta: \Delta \rightarrow G$, with $G$ isomorphic to either

$$
D_{6}=\left\langle t, p, s: t^{2}=p^{2}=s^{2}=(t p)^{3}=(p s)^{2}=(t s)^{2}=1\right\rangle=\Sigma_{3} \times \mathbf{Z}_{2}
$$

or

$$
\Sigma_{4}=\left\langle t, p, s: t^{2}=s^{2}=p^{2}=(t s)^{2}=(t p)^{3}=(s p)^{4}=1\right\rangle,
$$

$\operatorname{ker} \theta$ is a Fuchsian surface group and $X$ is conformally equivalent to $D / \theta^{-1}\langle t\rangle$.

The group $D_{6}$ in (8) is the dihedral group of 12 elements with a non-standart presentation.

Proof. Let $(X, f)$ be a non-cyclic trigonal surface, then $f: X \rightarrow \widehat{\mathrm{C}}$ is a 3 -sheeted branched covering. Let $B$ be the set of branch points of $f$. As in the proof of Theorem 2.3, the monodromy of $f$ is $\omega: \pi_{1}(\widehat{\mathrm{C}}-B) \rightarrow \Sigma_{3}$, where $\Sigma_{3}$ is the symmetric group of three elements $\{0,1,2\}$ and $f_{*}\left(\pi_{1}\left(X-f^{-1}(B)\right)\right)=$ $\omega^{-1}(\operatorname{Stab}(0))$. Then ker $\omega$ defines a regular covering $\widetilde{f}: \widetilde{X} \rightarrow \widehat{C}$, branched on $B$ and with automorphism group $\Sigma_{3}=\left\langle p, t: p^{2}=t^{2}=(t p)^{3}=1\right\rangle$. Therefore $\tilde{X} \rightarrow \tilde{X} /\langle t\rangle=X \stackrel{f}{\rightarrow} \tilde{X} / \Sigma_{3}=\widehat{C}$.

Before continuing the proof we prove the following

Lemma 2.7. The symmetry $\sigma$ lifts to a symmetry s of $\tilde{X}$.

Proof. To find a lift of $\sigma$ we begin by lifting $c$ by $\widetilde{X} \rightarrow \tilde{X} / \Sigma_{3}=\widehat{C}$. The branching points of $\widetilde{X} \rightarrow \widehat{C}$ have order 2 or 3 . Since the symmetry $\sigma$ is $1-1$ and $f \circ \sigma=c \circ f$, the branching points are sent by $c$ to branching points of the same order. If $\gamma \in \operatorname{ker} \omega$ then $\gamma$ lifts to three loops $\left\{\gamma_{i}: i=0,1,2\right\}$ in $X$. As $\sigma$ is 1-1 then $\left\{\sigma\left(\gamma_{i}\right)\right\}$ consists of three loops and $c \gamma \in \operatorname{ker} \omega$. Hence $c_{*}(\operatorname{ker} \omega)=\operatorname{ker} \omega$. Therefore $c$ can be lifted to $\widetilde{X}$. Let $s$ be a lift of $c$ that is also a lift of $\sigma$. Since $\widetilde{X} \rightarrow X$ is 2 -sheeted, $s$ has order 4 or 2 . Suppose that $s$ has order 4 . Then $s^{2}=t$, and using the uniqueness of $f$ given by [1] $s t p s=(t p)^{ \pm 1}$, so $s^{2} t p s^{2}=t p$. But using the presentation of $\Sigma_{3} s^{2} t p s^{2}=t t p t=p t$, that is a contradiction.

Now let $\widetilde{\Gamma}$ be a Fuchsian surface group uniformizing $\widetilde{X}$ and $\Gamma=\theta^{-1}(\langle t\rangle)$. Let $\widetilde{\sigma}$ be the lifting of $s, \rho$ the lifting of $p$ and $\tau$ the lifting of $t$ to $\mathscr{D}$ by $\mathscr{D} \rightarrow \mathscr{D} / \widetilde{\Gamma}$. We set $\Delta=\langle\widetilde{\Gamma}, \tau, \rho, \widetilde{\sigma}\rangle$ and $\widehat{\Delta}=\langle\widetilde{\Gamma}, \tau, \rho\rangle$ the universal covering transformations groups of $(\tilde{X},\langle t, p, s\rangle)$ and $\left(\tilde{X}, \Sigma_{3}\right)$. By construction $\Delta$ and $\widehat{\Delta}$ have signatures (7) and (5) respectively. Moreover, the group $G=\langle p, t, s\rangle \subset$ $\operatorname{Aut}(\widetilde{X})$ is a Coxeter group which contains commuting generating involutions $t$ and $s$. Thus $G$ is $D_{6}$ or $\Sigma_{4}$, and the order of $p s$ is 2 or 4 respectively. If $p s$ has order 2 , then $\widehat{\Delta}$ is the canonical Fuchsian subgroup of $\Delta$ and the orders of the ramification points are 3 or 2 . If $p s$ has order 4 , then the orders of the conic 
and corner points are 2,3 or 4 . The meromorphic function $\mathscr{D} / \Gamma \rightarrow \mathscr{D} / \widehat{\Delta}$ is the trigonal morphism. The action of $\Delta$ on the $\Gamma$-cosets gives us the required epimorphism $\theta: \Delta \rightarrow G$.

Assume now that we have an NEC group $\Delta$ with signature (7) and an epimorphism $\theta: \Delta \rightarrow G$, where $G$ is isomorphic to either $D_{6}$ with presentation (8) or $\Sigma_{4}$ with presentation (9), and $\operatorname{ker} \theta$ is a Fuchsian surface group. Let $X$ be the surface uniformized by $\Gamma=\theta^{-1}\langle t\rangle$. Thus $\Gamma$ is an index three non-normal subgroup of $\widehat{\Delta}=\theta^{-1}\langle p, t\rangle$, with signature $\left(g,\left[2^{u}\right]\right)$ such that $\mathscr{D} / \Gamma$ is conformally equivalent to $X$. The meromorphic function $f: \mathscr{D} / \Gamma \rightarrow \mathscr{D} / \widehat{\Delta}$ is a trigonal morphism and the quotient $X=\mathscr{D} / \Gamma \rightarrow \mathscr{D} / \theta^{-1}(\langle s, t\rangle)$ provides a symmetry $\sigma$ of $X$ in such a way that $(X, f, \sigma)$ is a real non-cyclic trigonal surface.

\section{Real cyclic trigonal surfaces}

A Riemann surface represented by an algebraic curve given by an equation of the form:

$$
y^{3}=\prod\left(x-a_{i}\right) \prod\left(x-b_{j}\right)^{2},
$$

where the coefficients of the polynomial $\prod\left(x-a_{i}\right) \prod\left(x-b_{j}\right)^{2}$ are real is an example of real cyclic trigonal surface.

The complex conjugation in the $x$-plane induces an involution on the above curve and the fixed point set is exactly the set of real points of the curve. A natural problem is to determine the possible number of connected components of such a set and the topological type of the action of the involution induced by conjugation. The following result gives the answer to such questions:

THEOREM 3.1. Let $(X, f, \sigma)$ be a real cyclic trigonal surface, with $X$ of genus $g \geq 5$. Then the possible species of $\sigma$ are: -1 for odd genus $g$ and +3 , +1 and -1 for even genus $g$.

Proof. By Theorem 2.5 there exists an NEC group $\Delta$ with signature

$$
\left(0,+,\left[3^{u}\right],\left\{\left(3^{v}\right)\right\}\right),
$$

with $2 u+v=g+2$ (by the Riemann-Hurwitz formula), and an epimorphism $\theta: \Delta \rightarrow G$, where $G$ is isomorphic to either $D_{3}=\left\langle r, s: r^{3}=s^{2}=r s r s=1\right\rangle$ or $\mathrm{Z}_{6}=\left\langle r, s: r^{3}=s^{2}=r s r^{-1} s=1\right\rangle$, and $X$ is conformally equivalent to $\mathscr{D} / \operatorname{ker} \theta$, with $\operatorname{ker} \theta$ a Fuchsian surface group. We consider two cases:

Case 1. $G=D_{3}=\left\langle r, s: r^{3}=s^{2}=r s r s=1\right\rangle$. The species of $\sigma$ is determined by the signature of the group $\theta^{-1}(\langle s\rangle)=\Lambda$ because $X /\langle\sigma\rangle=\mathscr{D} / \Lambda$. As all the link periods are odd, by [6], there is a unique conjugacy class of generating reflections in $\Lambda$, so the species of $\sigma$ is \pm 1 . Let us now study the sign. To determine the sign of the species of $\sigma$, we study the Schreier coset graph $\mathscr{G}$ of $\Lambda$ in $\Delta$ 
given by a canonical presentation of $\Delta$. Equivalently the Schreier coset graph of $\langle s\rangle$ in $D_{3}$ given by the image by the epimorphism $\theta$ of the canonical generators. The graph $\mathscr{G}$ consists of three vertices $\left\{0=\langle s\rangle, 1=r\langle s\rangle, 2=r^{2}\langle s\rangle\right\}$. The sign in the species of $\sigma$ is - if and only if there is a cycle (that is not a loop) in $\mathscr{G}$ with an odd number of edges produced by generators of $\Delta$ that are orientation reversing transformations, see Theorem 2 of [9]. This fact happens if and only if at least one of the following three conditions are satisfied:

1. The signature of $\Delta$ contains proper periods,

2. The epimorphism $\theta$ maps the connecting generator $e$ of $\Delta$ to an element different from the identity,

3. The generating reflections of $\Delta$ are mapped by $\theta$ on more than two involutions of $D_{3}$.

If one of the above properties is satisfied, either $x_{1}^{\varepsilon} c_{1}$ (for some power $\varepsilon$ ), $e^{\varepsilon} c_{1}$ or a product of three generating reflections of $\Delta$ mapped to three different involutions of $D_{3}$ provides the cycle in $\mathscr{G}$ telling us that the species is -1 .

If the conditions 1, 2 and 3 are not satisfied, then applying the RiemannHurwitz formula we have that the genus of the surface $X$ is even.

Now we shall prove the existence of the cyclic trigonal surfaces in the conditions of the Theorem for this case. Let $\Delta$ be an NEC group with signature $\left(0,+,\left[3^{2}\right],\left\{\left(3^{g-4}\right)\right\}\right)$. The following epimorphism

$$
\theta_{1}: \Delta \rightarrow\left\langle r, s: r^{3}=s^{2}=r s r s=1\right\rangle
$$

defined by:

$$
\begin{gathered}
\theta_{1}\left(x_{1}\right)=r, \theta_{1}\left(c_{1}\right)=s, \\
\theta_{1}\left(c_{2}\right)=s r, \ldots, \theta_{1}\left(c_{g-3}\right)=s \text { or } s r(\text { according to the parity of } g), \\
\theta_{1}(e)=r^{\varepsilon} \text { such that } \theta_{1}\left(c_{1} e^{-1} c_{g-3} e\right)=1, \\
\text { and } \theta_{1}\left(x_{2}\right)=r^{\mu} \text { such that } 1+\varepsilon+\mu \equiv 0(\bmod 3),
\end{gathered}
$$

provides, by Theorem 2.5 , a real cyclic trigonal surface with a symmetry of species -1 .

For even genera the signature $\left(0,+,[-],\left\{\left(3^{g+2}\right)\right\}\right)$ and the epimorphism

$$
\theta_{2}: \Delta \rightarrow\left\langle r, s: r^{3}=s^{2}=r s r s=1\right\rangle
$$

defined by:

$$
\theta_{2}\left(c_{1}\right)=s, \theta_{2}\left(c_{2}\right)=s r, \ldots, \theta_{2}\left(c_{g+3}\right)=s, \theta_{2}(e)=1
$$

yield, by Theorem 2.5, a real cyclic trigonal surface with a symmetry of species +1 . 
Case 2. $G=\mathrm{Z}_{6}=\left\langle r, s: r^{3}=s^{2}=r s r^{-1} s=1\right\rangle$. The species of $\sigma$ is determined by the signature of the group $\theta^{-1}(\langle s\rangle)=\Lambda$. In this case the NEC group $\Delta$ must have signature $\left(0,+,\left[3^{u}\right],\{(-)\}\right)$, with $2 u=g+2$, and then the surface $X$ must have even genus. The species of $\sigma$ is \pm 1 if $\theta(e) \neq 1$, and the species of $\sigma$ is \pm 3 if $\theta(e)=1$. Since the index of $\Lambda$ in $\Delta$ is three and so odd, the symmetry $\sigma$ has sign + in its species by Corollary 2 of [9]. Clearly there exists NEC groups with signature $\left(0,+,\left[3^{u}\right],\{(-)\}\right)$ and epimorphisms on $Z_{6}$ providing, by Theorem 2.5, real cyclic trigonal surfaces with both possible species, see the next example for genus $g=6$.

EXAMPLE 3.2. Consider an NEC group $\Delta$ with signature $\left(0,+,\left[3^{4}\right],\{(-)\}\right)$, and define epimorphisms $\theta_{1}, \theta_{2}: \Delta \rightarrow\left\langle r, s: r^{3}=s^{2}=r s r^{-1} s=1\right\rangle$ by

$$
\begin{gathered}
\theta_{1}\left(c_{1}\right)=s, \theta_{1}\left(x_{i}\right)=r, i=1, \ldots, 4, \theta_{1}(e)=r^{-1}, \text { and } \\
\theta_{2}\left(c_{1}\right)=s, \theta_{2}\left(x_{1}\right)=r, \theta_{2}\left(x_{2}\right)=r^{-1}, \theta_{2}\left(x_{3}\right)=r, \theta_{2}\left(x_{4}\right)=r^{-1}, \theta_{2}(e)=1 .
\end{gathered}
$$

The first epimorphism provides a separating symmetry on $\mathscr{D} / \operatorname{ker} \theta_{1}$ with one oval, and the second epimorphism gives a separating symmetry on $\mathscr{D} / \operatorname{ker} \theta_{2}$ with three ovals.

From the proof of the Theorem 3.1, we have:

Remark 3.3. Let $(X, f, \sigma)$ be a real cyclic trigonal surface, with $X$ of genus $g \geq 5$. Let $\varphi$ be a deck transformation of the covering $f$. If $X$ is represented by an algebraic curve given by an equation of the form $y^{3}=\prod\left(x-a_{i}\right) \prod\left(x-b_{j}\right)^{2}$ with real coefficients, then the group $G=\langle\varphi, \sigma\rangle$ is $D_{3}$. In this case the surface $X$ is included in Case 1 of the proof of Theorem 3.1. Furthermore Fix $(\sigma)$ is a closed simple curve and Fix $(\sigma)$ is a covering of degree one over $\mathbf{P}^{1}(\mathbf{R})$ in $\widehat{\mathrm{C}}$. If $G=\langle\varphi, \sigma\rangle$ is $\mathrm{Z}_{6}$ (Case 2 in the proof of Theorem 3.1) then Fix $(\sigma)$ consists in one or three closed curves covering with degree three or one over $P^{1}(R)$ respectively. Hence the real cyclic trigonal surfaces for this last case are represented by real trigonal curves of type (1) and (2) in the classification in page 179 of [7].

\section{Real non-cyclic trigonal surfaces}

A Riemann surface $X$ represented by a curve given by a polynomial equation with real coefficients of the form

$$
y^{3}+y b(x)+c(x)=0,
$$

and $b(x)$ not identically 0 is a real non-cyclic trigonal surface. 
The next theorem will tell us the possible species for the symmetry of the complex algebraic curve defined by the complex conjugation, so we know the number of connected components of the real part of the curve.

We recall the action of the elements of order 2 in $D_{6}$ with presentation (8) on the $\langle t, s\rangle$-cosets. Let $\delta: D_{6} \rightarrow \Sigma_{3}$ be the representation of the action of $D_{6}$ by left multiplication on

$$
D_{6} /\langle t, s\rangle=\{0,1,2\}=\left\{\langle t, s\rangle, t p\langle t, s\rangle,(t p)^{2}\langle t, s\rangle\right\},
$$

the set of $\langle t, s\rangle$-cosets. Then $\delta(t)=(1,2), \delta(p)=(0,2), \delta(s)=i d$.

Using the above action and the structure of NEC groups (see [8] and [13]), we have the following claims that will be useful in the proof of the next theorem:

Let $\Delta$ be an NEC group with signature $\left(0,+,[-],\left\{\left(2^{v}\right)\right\}\right)$ and let $\theta: \Delta \rightarrow$ $D_{6}$ be an epimorphism as in Theorem 2.6. The permutation $\delta(s)$ fixes all three $\langle t, s\rangle$-cosets and $\delta\left((t p)^{w} t s\right)$ fixes one $\langle t, s\rangle$-coset. The generating reflections of $\Lambda$ correspond to fixed $\langle t, s\rangle$-cosets. According to [8] there is a presentation of $\Lambda=\theta^{-1}(\langle s, t\rangle)$ such that each generating reflection $c_{j}$ of $\Delta$ gives rise to three generating reflections $c_{j 0}, c_{j 1}, c_{j 2}$ of $\Lambda$ if $\theta\left(c_{j}\right)=s$, and $c_{j}$ induces one generating reflection $c_{j k}$ if $\theta\left(c_{j}\right)=(t p)^{w} t s, \delta\left(\theta\left(c_{j}\right)\right)(k)=k$. Furthermore, as $c_{j} c_{j+1}$ and, therefore, $\theta\left(c_{j} c_{j+1}\right)$ have order two, then, given two consecutive generating reflections $c_{j}, c_{j+1}$, either $\theta\left(c_{j}\right)=s$ or $\theta\left(c_{j+1}\right)=s$. Now if $\delta\left(\theta\left(c_{j} c_{j+1}\right)\right)=(k)(m, n)$ and $\theta\left(c_{j}\right)=s$, then $c_{j m}$ is conjugated to $c_{j n}$ and $c_{j k}$ is conjugated to $c_{(j+1) k}$. If $\delta\left(\theta\left(c_{j} c_{j+1}\right)\right)=(k)(m, n)$ and $\theta\left(c_{j+1}\right)=s$, then $c_{j k}$ is conjugated to $c_{(j+1) k}$ and $c_{(j+1) m}$ is conjugated to $c_{(j+1) n}$. The index $j$ ranges in $Z_{v}$.

Theorem 4.1. Let $(X, f, \sigma)$ be a real non-cyclic trigonal surface, with $X$ of genus $g \geq 5$. Then there is no restriction to the species of $\sigma$ besides the ones given by Harnack's theorem.

Proof. Consider, as in Theorem 2.6, an NEC group $\Delta$ with signature

$$
\left(0,+,[-],\left\{\left(2^{2 g+4}\right)\right\}\right)
$$

and an epimorphism $\theta: \Delta \rightarrow D_{6}$ such that $\operatorname{ker} \theta$ is a Fuchsian surface group. By Theorem 2.6 the required real non-cyclic trigonal surface $X$ will be the Riemann surface uniformized by $\Gamma=\theta^{-1}(\langle t\rangle)$ defined in Theorem 2.6. As in Theorem 2.6, let $\Lambda=\theta^{-1}(\langle s, t\rangle)$. The surface $X$ admits a symmetry $\sigma$ given by the action of $\Lambda / \Gamma$ on $X$. We separate the proof in two cases according whether $\sigma$ separates or not. We shall construct epimorphisms $\theta$ providing all the possible species for $\sigma$.

Case 1. The species of $\sigma$ is $+k$, in this case $k \equiv g+1(\bmod 2)$. We define $\theta: \Delta \rightarrow D_{6}$ by $\theta\left(c_{2 m+1}\right)=s, 0 \leq m \leq g+1, \theta\left(c_{2 j}\right)=t s$, 


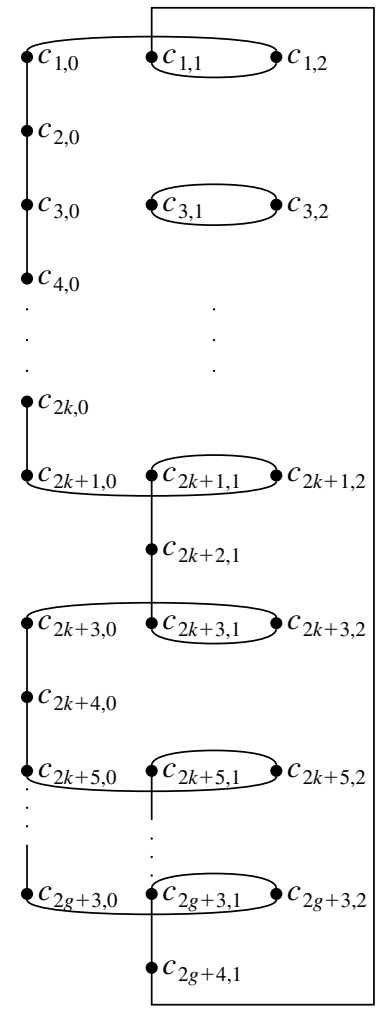

FIGURE 1

$0 \leq j \leq k, \theta\left(c_{2 k+4 l+2}\right)=(t p)^{2} t s, \theta\left(c_{2 k+4 l+4}\right)=t s, 0 \leq l \leq(g-1-k) / 2$, $\theta\left(c_{2 g+4}\right)=(t p)^{2} t s$.

First, we compute the number of ovals of $\sigma$, i.e. the number of conjugacy classes of generating reflections of $\Lambda$. By the algorithm above, each generating reflection $c_{2 m+1}$ induces three generating reflections $c_{2 m+1,0}, c_{2 m+1,1}, c_{2 m+1,2}$ of $\Lambda$; each generator $c_{2 j}$ induces the generator $c_{2 j, 0}$, each generator $c_{2 k+4 l+2}$ and $c_{2 g+4}$ gives rise to the generators $c_{2 k+4 l+2,1}$ and $c_{2 g+4,1}$. Finally each generator $c_{2 k+4 l+4}$ induces the generating reflection $c_{2 k+4 l+4,0}$ of $\Lambda$. Using the claims given before the statement of the theorem, we have that the conjugacy classes of reflections of $\Lambda$ correspond to the connected components of the graph $\mathscr{G}_{+}$given in Figure 1 whose vertices represent the above generating reflections of $\Lambda$. The graph $\mathscr{G}_{+}$consists of $k$ connected components, namely $C_{m}=\left\{c_{2 m+1,1}, c_{2 m+1,2}\right\}, 1 \leq m \leq k-1$ and $C_{k}=\mathscr{G}_{+}-\bigcup_{m=1}^{k-1} C_{m}$. Thus $\sigma$ has $k$ ovals.

To determine the sign of the species of $\sigma$, we describe the Schreier graph 
$\mathscr{G}$ of $\Lambda$ in $\Delta$, or equivalently the Schreier graph of $\langle s, t\rangle$ in $D_{6}$ induced by the epimorphism $\theta$. The graph $\mathscr{G}$ consists of three vertices $\{0,1,2\}$ and the edges which are not loops connect either 1 and 2, or 0 and 2. Then, the partition $\{0,1\} \cup\{2\}$ shows that $\mathscr{G}$ is bipartite and $\sigma$ separates, see Corollary 3 of [9]; note that all (non-trivial) generators of the canonical presentation of $\Delta$ are orientation reversing transformations (the connecting generator $e$ is in this case trivial).

Case 2. The species of $\sigma$ is $-k, 1 \leq k \leq g$. We define $\theta: \Delta \rightarrow D_{6}$ by

$$
\begin{gathered}
\theta\left(c_{2 m+1}\right)=s, 0 \leq m \leq g+1, \theta\left(c_{2 j}\right)=t s, \quad 0 \leq j \leq k, \\
\theta\left(c_{2 g+2}\right)=(t p) t s, \theta\left(c_{2 g+4}\right)=(t p)^{2} t s, \text { and } \\
\theta\left(c_{2 k+4 l+2}\right)=(t p)^{2} t s, \theta\left(c_{2 k+4 l+4}\right)=t s, \\
0 \leq l \leq(g-2-k) / 2 \text { for } g-k \equiv 0(\bmod 2), \text { and } \\
\theta\left(c_{2 k+4 l+2}\right)=(t p)^{2} t s, \theta\left(c_{2 k+4 l+4}\right)=t s, \\
0 \leq l \leq(g-3-k) / 2, \theta\left(c_{2 g}\right)=(t p)^{2} t s, \text { for } g-k \equiv 1(\bmod 2) .
\end{gathered}
$$

As in Case 1, each generating reflection $c_{2 m+1}$ induces three generators $c_{2 m+1,0}$, $c_{2 m+1,1}, c_{2 m+1,2}$, of $\Lambda$; each generator $c_{2 j}$ induces the generator $c_{2 j, 0}$, each generator $c_{2 k+4 l+2}$ and $c_{2 g+4}$ gives rise to the generators $c_{2 k+4 l+2,1}$ and $c_{2 g+4,1}$. Each generator $c_{2 k+4 l+4}$ induces the generator $c_{2 k+4 l+4,0}$. Finally the generator $c_{2 g+2}$ induces the generator $c_{2 g+2,2}$ of $\Lambda$. Again, to compute the number of ovals of $\sigma$, i.e. the number of conjugacy classes of reflections of $\Lambda$, we have to compute the number of connected components of the graph $\mathscr{G}_{-}$given in Figures 2 and 3 whose vertices represent the above generators $c_{i, j}$. The graph $\mathscr{G}_{-}$consists of $k$ connected components, namely $C_{m}=\left\{c_{2 m+1,1}, c_{2 m+1,2}\right\}$, $1 \leq m \leq k-1$ and $C_{k}=\mathscr{G}_{-}-\bigcup_{m=1}^{k-1} C_{m}$. Thus $\sigma$ has $k$ ovals.

To determine the sign of species of $\sigma$, we study the Schreier graph $\mathscr{G}$ of $\Lambda$ in $\Delta$, or equivalently the Schreier graph of $\langle s, t\rangle$ in $D_{6}$ induced by the epimorphism $\theta$. The graph $\mathscr{G}$ contains the following 3-cycle $0 \stackrel{(t p)^{2} t s}{\longrightarrow} 1 \stackrel{t s}{\longrightarrow}$ $2 \stackrel{(t p) t s}{\longrightarrow} 0$. Thus, $\mathscr{G}$ is not bipartite and $\sigma$ is non-separating.

Remark 4.2. The non-cyclic trigonal surfaces $(X, f, \sigma)$ constructed in the proof of the above theorem admit a symmetry $\sigma$ such that Fix $(\sigma)$ has a connected component having degree one over $\mathrm{P}^{1}(\mathrm{R})$, and the remaining components having degree zero over $P^{1}(R)$. These curves belong to type (4) of [7]. Hence with Theorem 4.1 we give the answer to a question of Gross and Harris.

The real trigonal curves of type (3) following the classification of [7] represent real non-cyclic trigonal surfaces: 


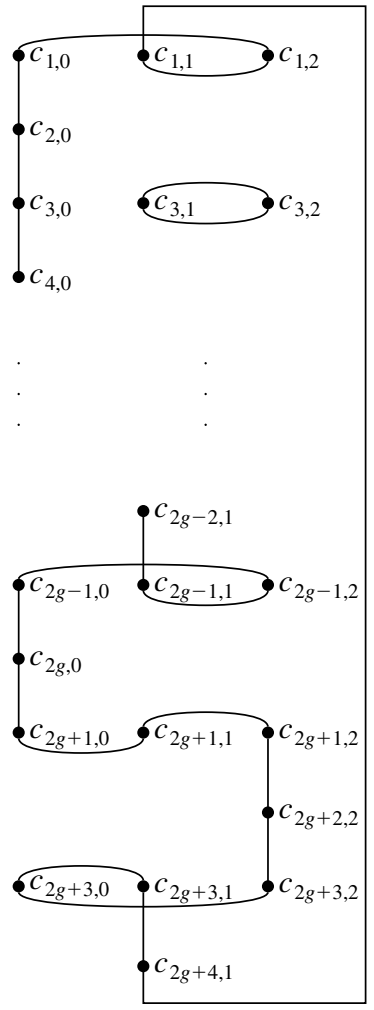

FIGURE 2. (case $g-k$ even)

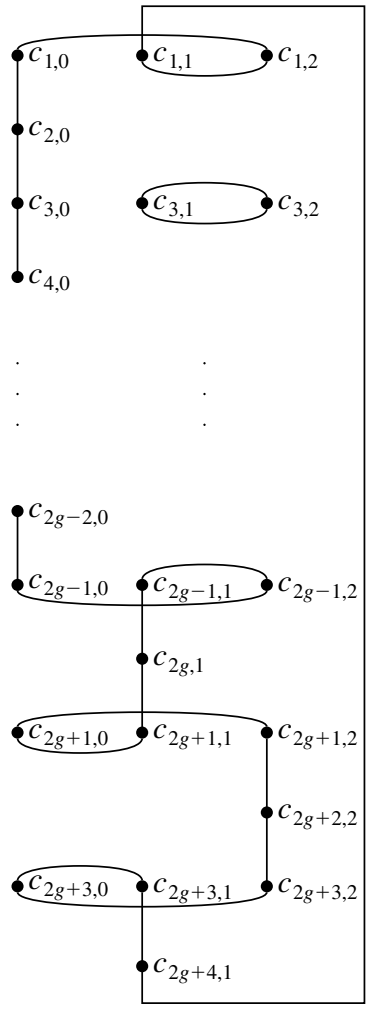

FIGURE 3. (case $g-k$ odd)

EXAMPLE 4.3. Consider an NEC group $\Delta$ with signature

$$
\left(0,+,\left[2^{g+2}\right],\{(-)\}\right),
$$

where $g \geq 5$ is odd, and an epimorphism $\theta: \Delta \rightarrow D_{6}$, defined by

$$
\begin{aligned}
& \theta(e)=t, \quad \theta\left(x_{i}\right)=p, \quad i=1, \ldots, g+1, \\
& \theta(c)=s, \quad \theta\left(x_{g+2}\right)=t,
\end{aligned}
$$

where $D_{6}$ has presentation (8). Then the real non-cyclic trigonal surface $X$ uniformized by $\Gamma=\theta^{-1}(\langle t\rangle)$ with symmetry $\sigma$ satisfies that $\operatorname{Fix}(\sigma)$ has two connected components, one of them of degree one over $P^{1}(R)$, and the other one of degree two over $\mathrm{P}^{1}(\mathrm{R})$. Hence $X$ is of type (3). 


\section{Antipodal trigonal surfaces}

Any symmetry of the Riemann sphere is conjugate to the complex conjugation or the antipodal map. There are trigonal Riemann surfaces $(X, f)$ admitting a symmetry $\sigma$ such that $\sigma$ induces the antipodal map $a$ on $\widehat{C}$ by $\mathscr{D} \rightarrow \mathscr{D} / \Gamma=$ $X \stackrel{f}{\rightarrow} \widehat{\mathrm{C}}$. In this case the symmetry $\sigma$ has no fixed points and the surface $X / \sigma$ has no boundary and it is non-orientable. These surfaces will be called antipodal trigonal surfaces, they correspond to real trigonal curves without real points.

With the same methods as in Theorem 2.5, it is easy to see that an antipodal cyclic trigonal surface is uniformized by a surface Fuchsian group $\Gamma=\operatorname{ker}(\theta)$, where $\theta$ is an epimorphism $\theta: \Delta \rightarrow G$, with $G$ isomorphic to $D_{3}$ or $\mathrm{Z}_{6}$ and $\Delta$ has signature

$$
\left(1,-,\left[3^{u}\right]\right)
$$

Note that $\mathscr{D} / \Delta$ is topologically the real projective plane.

Remark 5.1. Applying the Riemann Hurwitz formula and using the possible signatures (10) it is possible to show that antipodal cyclic trigonal surfaces must have even genera. And, by an argument as the used in Theorem 3.1, there are antipodal cyclic trigonal surfaces for any given even genus.

With the same procedure as in Theorem 2.6, it can be proved that an antipodal non-cyclic trigonal surface is uniformized by the Fuchsian group $\Gamma=\theta^{-1}\langle t\rangle$, for appropriate epimorphisms $\theta: \Delta \rightarrow G$, where $G=D_{6}$ with presentation (8) or $G=\Sigma_{4}$ with presentation (9) and $\Delta$ has signature

$$
\left(1,-,\left[2^{u_{1}}, 3^{u_{2}}, 4^{u_{3}}\right]\right)
$$

REMARK 5.2. By an argument as the used in Theorem 4.1, given an integer $g \geq 5$ it is possible to find an NEC group with signature $\left(1,-,\left[2^{v_{1}}, 3^{v_{2}}\right]\right)$ and an epimorphism $\theta: \Delta \rightarrow D_{6}$, such that the Fuchsian group $\theta^{-1}\langle t\rangle$ uniformizes an antipodal non-cyclic trigonal surface of genus $g$.

\section{REFERENCES}

1. Accola, R. D. M., Strongly branched coverings of closed Riemann surfaces, Proc. Amer. Math. Soc.26 (1970), 315-322.

2. Accola, R. D. M., On cyclic trigonal Riemann surfaces, I, Trans. Amer. Math. Soc. 283 (1984), 423-449.

3. Accola, R. D. M., A classification of trigonal Riemann surfaces, Kodai Math. J. 23 (2000), $81-87$. 
4. Bujalance, E., Costa, A. F., A combinatorial approach to the symmetries of $M$ and M-1 Riemann surfaces, in Discrete Groups and Geometry (eds. W. J. Harvey and C. Maclachlan), London Math. Soc. Lecture Note Ser. 173 (1992), 16-25.

5. Bujalance, E., Costa, A. F., On symmetries of p-hyperelliptic Riemann surfaces, Math. Ann. 308 (1997), 31-45.

6. Bujalance, E., Costa, A. F., Singerman, D., Application of Hoare's theorem to symmetries of Riemann surfaces, Ann. Acad. Scien. Fenn. 18 (1993), 307-322.

7. Gross, B. H., Harris, J., Real algebraic Curves, Ann. Sci. École Norm. Sup. 14 (1981), $157-182$.

8. Hoare, A. H. M., Subgroups of NEC groups and finite permutation groups, Quart. J. Math. 41 (1990), 45-59.

9. Hoare, A. H. M., Singerman, D., The orientability of subgroups of plane groups, Proc. of Groups, St. Andrews, 1981, London Math. Soc. Lecture Note Ser. 71 (1981), 221-227.

10. Klein, F., Über Realitätsverhältnisse bei einem beliebigeen Geschlechte zugehörigen Normalkurven der $\varphi$, Math. Ann. 42 (1893), 1-29.

11. Kato, T., Horiuchi, R., Weirstrass gap sequences at the ramification points of trigonal Riemann surfaces, J. Pure Appl. Alg. 50 (1988), 271-285.

12. Seifert, H., Threlfall, W., A Textbook of Topology, Academic Press, New York 1980.

13. Singerman, D., On the structure of non-Euclidean crystallographic groups, Math. Proc. Cambridge Philos. Soc. 76 (1974), 233-240.

DEPTO. DE MATEMÁTICAS FUNDAMENTALES

UNED

C/ SENDA DEL REY 9

28040 MADRID

SPAIN

E-mail: acosta@mat.uned.es
DEPARTMENT OF MATHEMATICS LINKÖPING UNIVERSITET

58183 LINKÖPING

SWEDEN

E-mail:miizq@mai.liu.se 